\title{
Increased expression of NFATc1 in giant cell lesions of the jaws, cherubism and brown tumor of hyperparathyroidism
}

\author{
ALESSANDRA PIRES DUARTE ${ }^{1}$, CAROLINA CAVALIÉRI GOMES ${ }^{2}$, \\ RICARDO SANTIAGO GOMEZ ${ }^{1}$ and FABRÍCIO REZENDE AMARAL ${ }^{1}$
}

Departments of ${ }^{1}$ Oral Surgery and Pathology and ${ }^{2}$ Pathology, Universidade Federal de Minas Gerais, Av. Antonio Carlos, 6627, Belo Horizonte-MG, CEP 31270 901, Brazil

Received December 15, 2010; Accepted March 15, 2011

DOI: $10.3892 / \mathrm{ol} .2011 .274$

\begin{abstract}
A variety of diseases of the jaws may present multinucleated giant cells. These diseases include central giant cell lesions (CGCL), peripheral giant cell lesions (PGCL), brown tumor of hyperparathyroidism (BTH), and cherubism. The multinucleated giant cells in these lesions are osteoclast-like. Since NFATc1 plays a significant role in osteoclast differentiation, the present study aimed to compare the expression of NFATc1 in CGCL, PGCL, BTH and cherubism. A total of 14 formalin-fixed and paraffin-embedded tissue samples of CGCL $(n=4)$, PGCL $(n=5)$, BTH $(n=3)$ and cherubism $(n=2)$ were included in the study. An immunohistochemical analysis was performed to investigate the NFATc1 protein. The majority of giant cells in all of the cases were positive for nuclear NFATc1 and the immunostaining pattern was similar in all of the groups. Although our study supports the hypothesis that giant cell accumulation in PGCL, CGCL, BTH and cherubism is mediated by NFATc1, functional studies are required to investigate this hypothesis.
\end{abstract}

\section{Introduction}

Various conditions of the jaws may present multinucleated giant cells. These include central giant cell lesions (CGCL), peripheral giant cell lesions (PGCL), brown tumor of hyperparathyroidism (BTH), and cherubism. These lesions show multinucleated osteoclast-like giant cells in a background of oval to spindle-shaped mononuclear cells.

PGCL is a reactive lesion usually associated with local irritating factors in gingiva (1). CGCL is an intra-osseous lesion of unknown etiology that occurs mainly in the mandible of patients ranging from 10 to 25 years (2). Cherubism is an autosomal-dominant genetic disease that affects the jaws (3).

Correspondence to: Dr Fabrício Rezende Amaral, Department of Oral Surgery and Pathology, School of Dentistry, Universidade Federal de Minas Gerais, Av. Antonio Carlos, 6627, Belo Horizonte-MG, CEP 31270 901, Brazil

E-mail: framaral2@ig.com.br

Key words: giant cell lesions, NFATc1, cherubism, brown tumor
The disease is characterized by bilateral expansion of the maxilla and/or mandible, usually detected in early childhood, and shows progressive growth until puberty (4). BTH is a nonneoplastic lesion resulting from abnormal bone metabolism in hyperparathyroidism and may be associated with the primary or secondary types of the disease (5).

The gene mutated in cherubism has been mapped to chromosome 4p16.3 (6). Missense mutations were observed in the $S H 3 B P 2$ gene mainly clustered in exon 9, which encodes a proline-rich region of the protein $(7,8)$. Recently, a somatic mutation of this gene was described in a case of CGCL (9).

NFATc1 plays a significant role in osteoclast differentiation. This protein is considered to be the osteoclastogenesis master transcription factor. NFATc1 is located in cytoplasm and is activated after RANKL signaling in osteoclast precursor cells (10). Downstream stimulation by RANKL promotes the formation of a complex containing a second messenger, SH3BP2, which results in the upregulation of intracellular calcium (11). Increased levels of calcium promote the displacement of NFATc1 to the nucleus where it binds to its own promoter. This binding leads to the autoamplification of NFATc1 and activation of osteoclasteogenesis-specific genes. In lesions with the $S H 3 B P 2$ mutant, as in the case of cherubism, upregulation of the calcium promotes a constant displacement of NFATc1 to the nucleus and a subsequent increased osteoclast differentiation (12).

Recently an increased transcription of the NFATc1 in was found in giant cell lesions (13). The present study aimed to compare the expression of NFATc1 in CGCL, PGCL, BTH and cherubism.

\section{Materials and methods}

A total of 14 formalin-fixed and paraffin-embedded tissue samples of PGCL $(n=5)$, CGCL $(n=4)$, BTH $(n=3)$ and cherubism $(n=2)$ were included in the present study. Table I shows the age, gender and tumor location in each group.

The immunohistochemistry protocols used are described elsewhere (13). Briefly, paraffin-embedded tissue sections were incubated with NFATc1 antiserum (diluted 1:50, Clone 7A6, SantaCruz Biotechnology Inc., Santa Cruz, CA, USA). The immunohistochemical staining was performed using a highly sensitive polymer-based system (EnVision, 
Table I. Clinical data and nuclear immunohistochemical expression of NFATc1.

\begin{tabular}{|c|c|c|c|c|c|}
\hline Lesion & Age & Gender & Location & $\%$ positive giant cells & $\%$ positive mononuclear cells \\
\hline \multicolumn{6}{|l|}{ CGCL } \\
\hline 1 & 19 & $\mathrm{M}$ & Mand. & 100.0 & 8.1 \\
\hline 2 & 16 & $\mathrm{~F}$ & Mand. & 93.8 & 4.7 \\
\hline 3 & 15 & $\mathrm{~F}$ & Max. & 96.3 & 16.7 \\
\hline 4 & 12 & $\mathrm{~F}$ & Max. & 91.0 & 9.0 \\
\hline \multicolumn{6}{|l|}{ PGCL } \\
\hline 1 & 7 & $\mathrm{~F}$ & Mand. & 93.0 & 2.4 \\
\hline 2 & 11 & $\mathrm{M}$ & Max. & 98.2 & 8.9 \\
\hline 3 & 37 & $\mathrm{~F}$ & Mand. & 100.0 & 7.5 \\
\hline 4 & 16 & $\mathrm{M}$ & Mand. & 94.6 & 5.4 \\
\hline 5 & 22 & M & Max. & 78.0 & 3.3 \\
\hline \multicolumn{6}{|l|}{ BTH } \\
\hline 1 & 43 & $\mathrm{~F}$ & Mand. & 95.5 & 8 \\
\hline 2 & 39 & $\mathrm{M}$ & Mand. & 90.8 & 6.9 \\
\hline 3 & 53 & $\mathrm{~F}$ & Max. & 99.2 & 15.3 \\
\hline \multicolumn{6}{|c|}{ Cherubism } \\
\hline 1 & 15 & M & Mand. & 87.3 & 4.1 \\
\hline 2 & 16 & $\mathrm{M}$ & Mand. & 93.0 & 8.9 \\
\hline
\end{tabular}

CGCL, central giant cell lesion; PGCL, peripheral giant cell lesion; BTH, brown tumor of hyperparathyroidism; M, male; F, female; Mand., mandible and Max., maxilla.
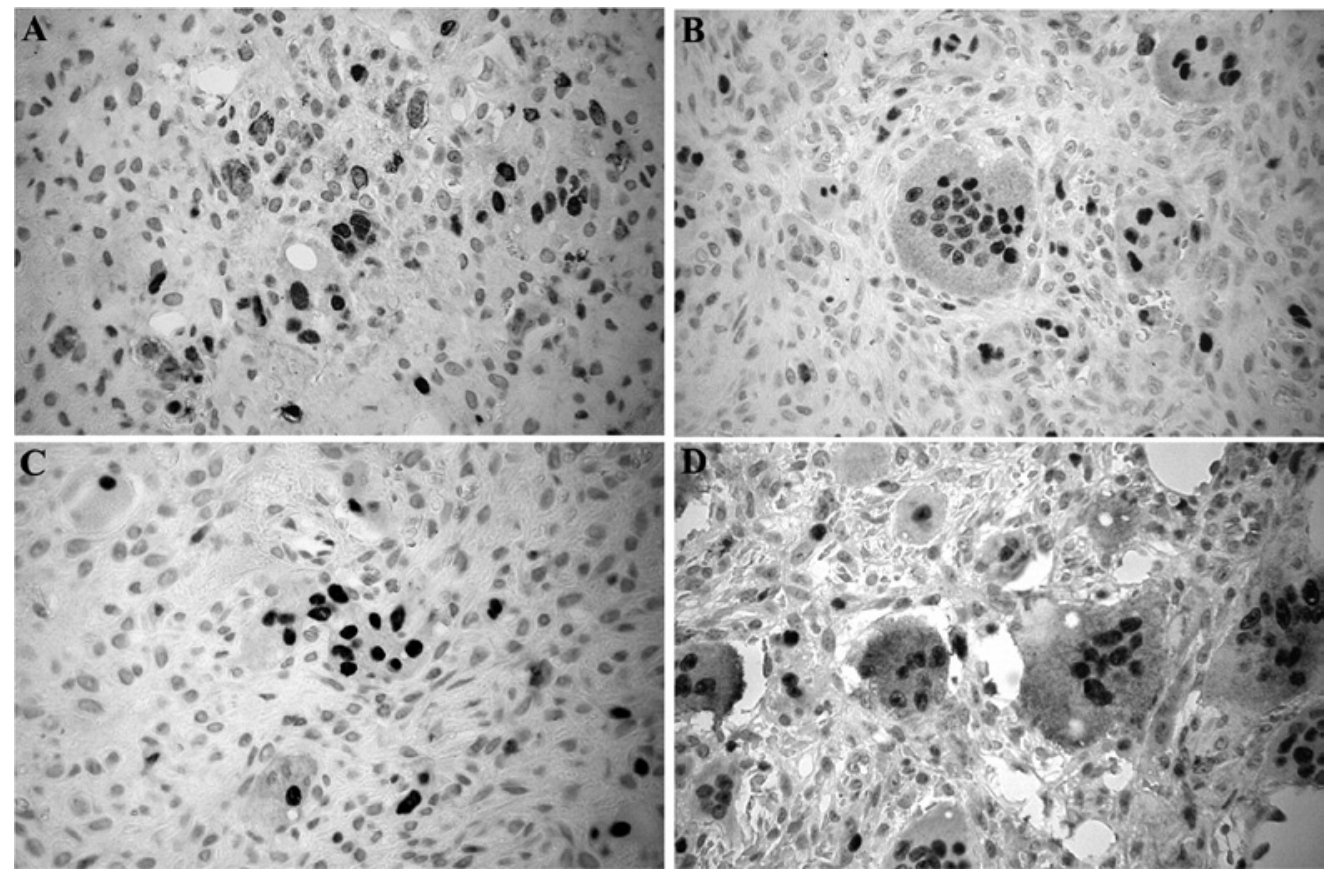

Figure 1. Immunohistochemical expression of NFATc1 in (A) central giant cell lesion, (B) peripheral giant cell lesion, (C) brown tumor of hyperparathyroidism and (D) cherubism (original magnification, $\mathrm{x} 400$ ).

Dako Corporation, Carpinteria, CA, USA) with the diaminobenzidine substrate solution as chromogen (Sigma, St. Louis, MO, USA). The sections were counterstained with hematoxylin.

Only sections containing sufficient tumor cells used to assess the antibody reactivity were included in the investigation. Two experienced pathologists made an independent analysis of each case, regardless of staining intensity. Six high-power fields (magnification, x400) were examined and the percentage of mononuclear and multinucleated positively stained cells was obtained for each case. Only nuclear immunoexpression was evaluated. 


\section{Results}

The imunohistochemical profile of NFATc1 in giant cell lesions, BTH and cherubism are shown in Table I. It was found that most of the giant cells in all of the cases were positive for nuclear NFATc1. Less than $17 \%$ of the mononuclear ovoid and spindle-shaped cells were immunopositive for this protein. Fig. 1 shows the immunostaining pattern of NFATc1 in each lesion group. The immunostaining pattern was similar in all lesions.

\section{Discussion}

Increased levels of NFATc1 in bone marrow cells play an important role in osteoclastogenesis. Previous studies showed that the giant cells found in PGCL, CGCL and cherubism exhibit osteoclast-like characteristics (14). NFATc1 is a cytoplasmic protein activated by calcineurin, $\mathrm{Ca}^{+} /$calmodulindependent protein phosphatase. This activation increases the levels of calcium, leading to NFATc1 translocation to the nucleus where it binds to its own promoter. After RANKL signaling, a complex containing a SH3BP2 second messenger is involved in a calcineurin cascade that activates NFATc1, increasing osteoclastogenesis and giant cell formation (11). $S H 3 B P 2$ is mutated in cherubism, and it was further demonstrated that this gene is also mutated in CGCL (9).

Results of our previous study showed an increased transcription of NFATc1 together with the positive immunostaining of this protein mainly in the nucleus of the multinucleated giant cell lesions of the jaws (13). In the present study, the immunoexpression of NFATc1 was compared in different lesions containing multinucleated giant cells. Most of the giant cells in all of the lesions exhibited NFATc1-positive nuclear staining. This finding supports the hypothesis that giant cell accumulation in PGCL, CGCL, BTH and cherubism is mediated by NFATc1. However, studies are required to examine whether NFATc1 modulation is an alternative molecular tool in the prevention or regulation of the growth of these lesions.

Apart from the important role in osteoclastogenesis, NFAT is crucial for thymocyte survival (15). This protein forms a complex with the protein AP-1 to bind in specific DNA sites. Loss of NFAT:AP-1 complex activity in the nucleus promotes apoptosis of these cells (16). Glucocorticoids impair this complex formation and induce apoptosis of the immature thymocytes (17). Corticosteroids have been used successfully in the treatment of certain cases of giant cell lesions of the jaws (18-20). A previous study showed that glucocorticoids inhibit osteoclast precursor proliferation (21). Findings of this study showed that glucocorticoids disrupt osteoclasts in the cytoskeleton and consequently affect the function of these cells, decreasing bone resorption. Further studies are required to demonstrate whether multinucleated giant cell formation is inhibited by corticosteroids via modulation of the NFAT pathway.

\section{Acknowledgements}

This study was supported by grants from Conselho Nacional de Desenvolvimento Científico e Tecnológico $(\mathrm{CNPq})$ and Fundação de Amparo à Pesquisa do Estado de
Minas Gerais (FAPEMIG), Brazil. Dr RS Gomez is a research fellow of CNPq.

\section{References}

1. Katsikeris N, Kakarantza-Angelopoulou E and Angelopoulos AP: Peripheral giant cell granuloma. Clinicopathologic study of 224 new cases and review of 956 reported cases. Int J Oral Maxillofac Surg 17: 94-99, 1998.

2. De Lange J, van den Akker HP and van den Berg H: Central giant cell granuloma of the jaw: a review of the literature with emphasis on therapy options. Oral Surg Oral Med Oral Pathol Oral Radiol Endod 104: 603-615, 2007.

3. Von Wowern N: Cherubism: a 36-year long-term follow-up of 2 generations in different families and review of the literature. Oral Surg Oral Med Oral Pathol Oral Radiol Endod 90: 765-772, 2000.

4. Hyckel P, Berndt A, Schleier P, Clement JH, Beensen V, Peters H and Kosmehl: Cherubism - new hypotheses on pathogenesis and therapeutic consequences. J Craniomaxillofac Surg 33: 61-68, 2005.

5. Pecovnik Balon B and Kavalar R: Brown tumor in association with secondary hyperparathyroidism. A case report and review of the literature. Am J Nephrol 18: 460-463, 1998.

6. Bell SM, Shaw M, Jou YS, Myers RM and Knowles MA: Identification and characterization of the human homologue of $\mathrm{SH} 3 \mathrm{BP} 2$, an $\mathrm{SH} 3$ binding domain protein within a common region of deletion at $4 \mathrm{p} 16.3$ involved in bladder cancer. Genomics 44: 163-170, 1997.

7. Ueki Y, Tiziani V, Santanna C, et al: Mutations in the gene encoding c-Abl-binding protein SH3BP2 cause cherubism. Nat Genet 28: 125-126, 2001.

8. De Lange J, van Maarle MC, van den Akker HP and Redeker EJ: A new mutation in the SH3BP2 gene showing reduced penetrance in a family affected with cherubism. Oral Surg Oral Med Oral Pathol Oral Radiol Endod 103: 378-381, 2007.

9. Carvalho VM, Perdigao PF, Amaral FR, De Souza PE, De Marco L and Gomez RS: Novel mutations in the SH3BP2 gene associated with sporadic central giant cell lesions and cherubism. Oral Dis 15: 106-110, 2009.

10. Asagiri $\mathrm{M}$ and Takayanagi $\mathrm{H}$ : The molecular understanding of osteoclast differentiation. Bone 40: 251-264, 2007.

11. Lietman SA, Yin L and Levine MA: SH3BP2 is an activator of NFAT activity and osteoclastogenesis. Biochem Biophys Res Commun 371: 644-648, 2008.

12. Novack DV and Faccio R: Jawing about TNF: new hope for cherubism. Cell 128: 15-17, 2007.

13. Amaral FR, Brito JA, Perdigao PF, et al: NFATc1 and TNF alpha expression in giant cell lesions of the jaws. J Oral Pathol Med 39: 269-274, 2010.

14. Itonaga I, Hussein I, Kudo O, Sabokbar A, Watt-Smith S, Ferguson D and Athanasou NA: Cellular mechanisms of osteoclast formation and lacunar resorption in giant cell granuloma of the jaw. J Oral Pathol Med 32: 224-231, 2003.

15. Adachi S, Amasaki $\mathrm{Y}$, Miyatake S, Arai $\mathrm{N}$ and Iwata $\mathrm{M}$ : Successive expression and activation of NFAT family members during thymocyte differentiation. J Biol Chem 275: 14708-14716, 2000.

16. Wisniewska M, Stanczyk M, Grzelakowska-Sztabert B and Kaminska B: Nuclear factor of activated T cells (NFAT) is a possible target for dexamethasone in thymocyte apoptosis. Cell Biol Int 21: 127-132, 1997.

17. Wisniewska M, Pyrzynska B and Kaminska B: Impaired AP-1 dimers and NFAT complex formation in immature thymocytes during in vivo glucocorticoid-induced apoptosis. Cell Biol Int 28: 773-780, 2004.

18. Abdo EN, Alves LC, Rodrigues AS, Mesquita RA and Gomez RS: Treatment of a central giant cell granuloma with intralesional corticosteroid. Br J Oral Maxillofac Surg 43: 74-76, 2005.

19. Carlos R and Sedano HO: Intralesional corticosteroids as an alternative treatment for central giant cell granuloma. Oral Surg Oral Med Oral Pathol Oral Radiol Endod 93: 161-166, 2002.

20. Adornato MC and Paticoff KA: Intralesional corticosteroid injection for treatment of central giant-cell granuloma. J Am Dent Assoc 132: 186-190, 2001.

21. Kim HJ, Zhao H, Kitaura H, et al: Glucocorticoids and the osteoclast. Ann N Y Acad Sci 1116: 335-339, 2007. 